\title{
Isolation of Novel Mycobacterium Species from Skin Infection in an Immunocompromised Person
}

\author{
You-Ming Mei, ${ }^{1}$ Qian Zhang, ${ }^{1}$ Wen-Yue Zhang, ${ }^{1}$ Hai-Qin Jiang, Ying Shi, \\ Jing-Shu Xiong, Le Wang, Yan-Qing Chen, Si-Yu Long, Chun Pan, Gai Ge, \\ Zhen-Zhen Wang, Zi-Wei Wu, Yan Wang, Yi-Qun Jiang, Hong-Sheng Wang
}

We investigated a case of cutaneous infection in an immunocompromised patient in China that was caused by a novel species within the Mycobacterium gordonae complex. Results of whole-genome sequencing indicated that some strains considered to be $M$. gordonae complex are actually polyphyletic and should be designated as closely related species.

$M$ ycobacterium gordonae was first described 50 years ago as a slow-growing scotochromogenic nontuberculous mycobacterium. Previous research revealed vague molecular typing results for $M$. gordonae-like strains. For example, the RNA polymerase- $\beta$ (rpoB) PCR restriction analysis discriminates M. gordonae into 4 clusters even though cluster D does not hybridize well with the type strain (1). Two novel species, M. paragordonae and $M$. vicinigordonae, share $>99 \% 16$ S rRNA gene similarity with M. gordonae, which might also lead to confusion about their classification $(2,3)$.

M. gordonae is frequently isolated from water systems and clinical samples as a potential opportunistic pathogen (4,5); clinical infections ranging from skin and lung infections to disseminated systemic infections have been reported, especially in immunosuppressed patients $(6,7)$. Both the $M$. paragordonae and $M$. vicinigordonae strains were first isolated as nonpathogenic organisms from pneumonia patients $(2,3)$. $M$. paragordonae is often isolated from hospital water systems and devices, but only 1 case of iatrogenic $M$. paragordonae infection has been reported $(8,9)$. These reports reveal the dissimilar effects produced by different $M$. gordonae-like strains.

\footnotetext{
Author affiliation: Chinese Academy of Medical Sciences and Peking Union Medical College, Institute of Dermatology, Nanjing, China
}

DOI: https://doi.org/10.3201/eid2711.210426
The advent of whole-genome sequencing has brought genomewide analyses into common use to delineate species (10-12). The widely accepted cutoffs adopted for the average nucleotide identity (ANI) and in silico DNA-DNA hybridization (isDDH), $95 \%-97 \%$ for ANI and 70\% for isDDH, strongly correlate with traditional DDH division values, providing more robust resolution than phenotyping or mycolic acid analysis for determining mycobacterial taxonomy $(11,12)$. We report a case of cutaneous infection in Jiangsu Province, China, caused by a previously undescribed novel species belonging to the M. gordonae group.

\section{The Study}

A 63-year-old man was admitted to the hospital for a 5-year history of a nodule on his left shin. The asymptomatic lesion initially appeared as a papule and gradually developed into a dull red verrucous nodule with scales (Figure 1, panel A). No trauma history before the onset was reported. The patient had received a diagnosis of lupus erythematosus 30 years earlier and had taken oral prednisone $(20 \mathrm{mg} / \mathrm{d})$ over the previous year. Laboratory test results indicated no remarkable findings. Histologic examination of a skin sample showed irregular epithelial hyperplasia and granulomatous infiltrations of a large number of epithelioid histocytes, neutrophil cells, plasma cells, and lymphocytes in the dermal layer. After 19 days of culture, orange colonies were observed on modified Löwenstein-Jensen slants at $32^{\circ} \mathrm{C}$ (Figure 1, panel B). The organism was scotochromogenic with a smooth appearance and grew well at $32^{\circ} \mathrm{C}$ and $37^{\circ} \mathrm{C}$ on both Löwenstein-Jensen slants and Middlebrook 7H9 with oleic acid dextrose citrate. The colonies were confirmed to be rod-shaped, acid-fast bacterium.

\footnotetext{
${ }^{1}$ These first authors contributed equally to this article.
} 
We extracted DNA from the colonies for PCR analysis and compared the sequences using BLAST (https://blast.ncbi.nlm.nih.gov/Blast.cgi). The 16S rRNA (1452 bp) gene shared greatest similarity (99.51\%) with M. gordonae ASCr-1.2; gene sequencing showed rроB (365 bp) shared $97.53 \%$ and hsp65 (765 bp) $95.53 \%$ similarity with $M$. paragordonae 49061 . On the basis of these results, we diagnosed infection with a member of the M. gordonae complex. Drug sensitivity analysis revealed the bacterium to be sensitive to moxifloxacin, levofloxacin, ethambutol, and amikacin and resistant to clarithromycin, isoniazid, and rifampicin; we therefore prescribed a moxifloxacin regimen for the patient. Because the lesion had not healed over several years, we surgically resected it and applied photodynamic therapy after 2 months of antimicrobial drug treatment. One month later, the patient reported that the lesion had recovered well and refused further oral antimicrobial drugs. No recurrence was observed in the following year.

To accurately identify the pathogen to the species level, we performed whole-genome sequencing $(8,509,558$ reads, $110 \times)$ of the isolate X7091 using the Illumina Hiseq 4000 (https://www.illumina.com) and PacBio RS II (https:/ / www.pacb.com) platforms at the Beijing Genomics Institute. Sequence data indicated a 7.1-Mb genome $(7,319,570 \mathrm{bp})$ including a plasmid $(216,348 \mathrm{bp})$ with a guanine-cytosine content of $64.6 \%$ (Genbank accession no. GCA_017086405.1). The complete genome had a guanine-cytosine content of $66.7 \%$, similar to $M$. gordonae $(66.8 \%)$ and $M$. paragordonae $(67.0 \%)$. Functional annotation obtained through multiple databases revealed 6,704 coding sequences, 48 tRNA, 3 rRNA, and 35 small RNA genes.

We compared this isolate with all available genomes of the $M$. gordonae group using whole-genome-based computational strategies. ANI calculated by FastANI (https://github.com/ParBLiSS/ FastANI) revealed that the closest matches, with $M$. gordonae HMC_M15 (87.80\%) and M. gordonae DSM $44160(87.79 \%)$, were well below the threshold for species delineation (Appendix Table, https://wwwnc.cdc.gov/EID/article/27/11/21-0426-App1.pdf) (11). Evaluating isDDH using the Type Strain Genome Server (https://tygs.dsmz.de) showed weak relations with $M$. gordonae DSM 44160 (34.5\%) and $M$. paragordonae 49061 (31.3\%) (13) (Appendix Table); we found no closely related genome in the database. For M. paragordonae strains, ANI was $97.8 \%-98.6 \%$ and isDDH 80.4\%-99.9\%; for M. gordonae strains, ANI was $99.1 \%-99.9 \%$ and isDDH $93.0 \%-99.3 \%$.

The core-genome phylogeny of the M. gordonae complex, constructed using a previously described

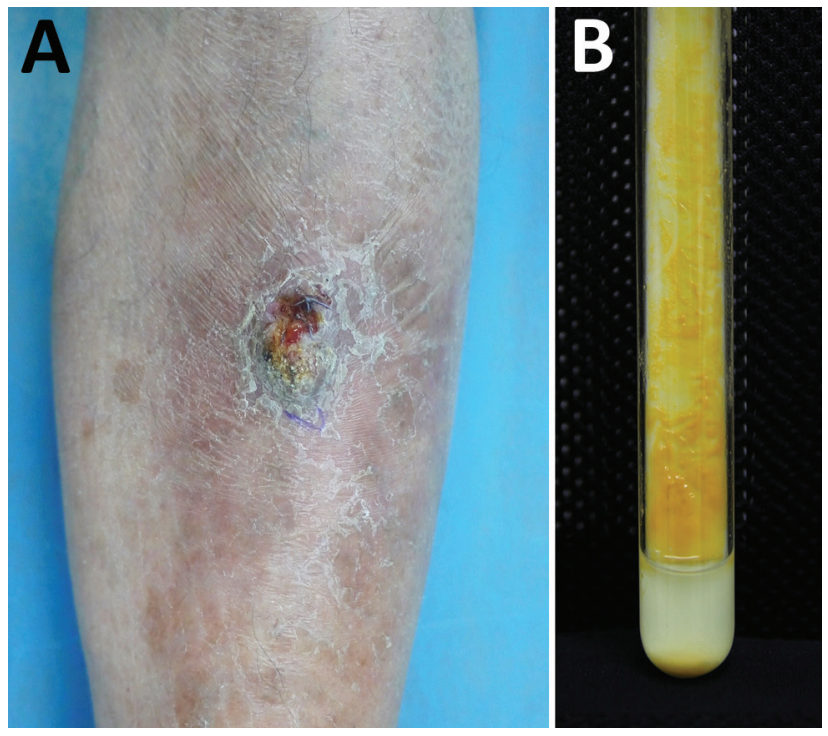

Figure 1. Novel Mycobacterium gordonae-like infection in a 63-year-old man in China. A) Verrucous dull nodule on the left shin of the patient. B) Mycobacterium colonies grown on LöwensteinJensen medium.

method, suggested that the isolated strain is a branch within the cluster but distant from other M. gordonaelike strains $(14,15)$ (Figure 2, panel B). Integration of these highly concordant results strongly suggested that the isolate is distinct from present M. gordonaelike strains and represents a novel species within the M. gordonae complex. We proposed Mycobacterium camsnse sp. nov. as the name for this strain.

When comparing the similarity index of all available genomes of the $M$. gordonae group, we found clear demarcations among the M. camsnse X7091, M. gordonae CTRI 14-8773, M. vicinigordonae 24, 7 M. paragordonae, and $3 \mathrm{M}$. gordonae strains including the type strain DSM 44160 (Figure 2). Two strains recorded as $M$. gordonae ssp. in the cluster of $M$. paragordonae may have previously been misclassified. M. gordonae CTRI 14-8773, isolated in Russia, also represents a novel species of the $M$. gordonae group. These results confirmed the genomic diversity of $M$. gordonae-like strains, corroborating that the M. gordonae group is polyphyletic and should be divided into $\geq 5$ closely related species.

\section{Conclusions}

M. gordonae is generally considered a minimally pathogenic mycobacteria. Nonetheless, clinical infections have been reported, even in immunocompetent individuals $(6,7)$. We isolated a distinct strain within the $M$. gordonae group from a skin infection using whole genome-level approaches based on ANI, is$\mathrm{DDH}$, and core gene phylogeny. We proposed the 
name Mycobacterium camsnse sp. nov. for this strain. 2 strains potentially misclassified as M. gordonae ssp., We also provided genomic insights into the heteroge- and demonstrated that the present $M$. gordonae group neity of the M. gordonae-like strains, including finding should be designated as 5 closely related species.

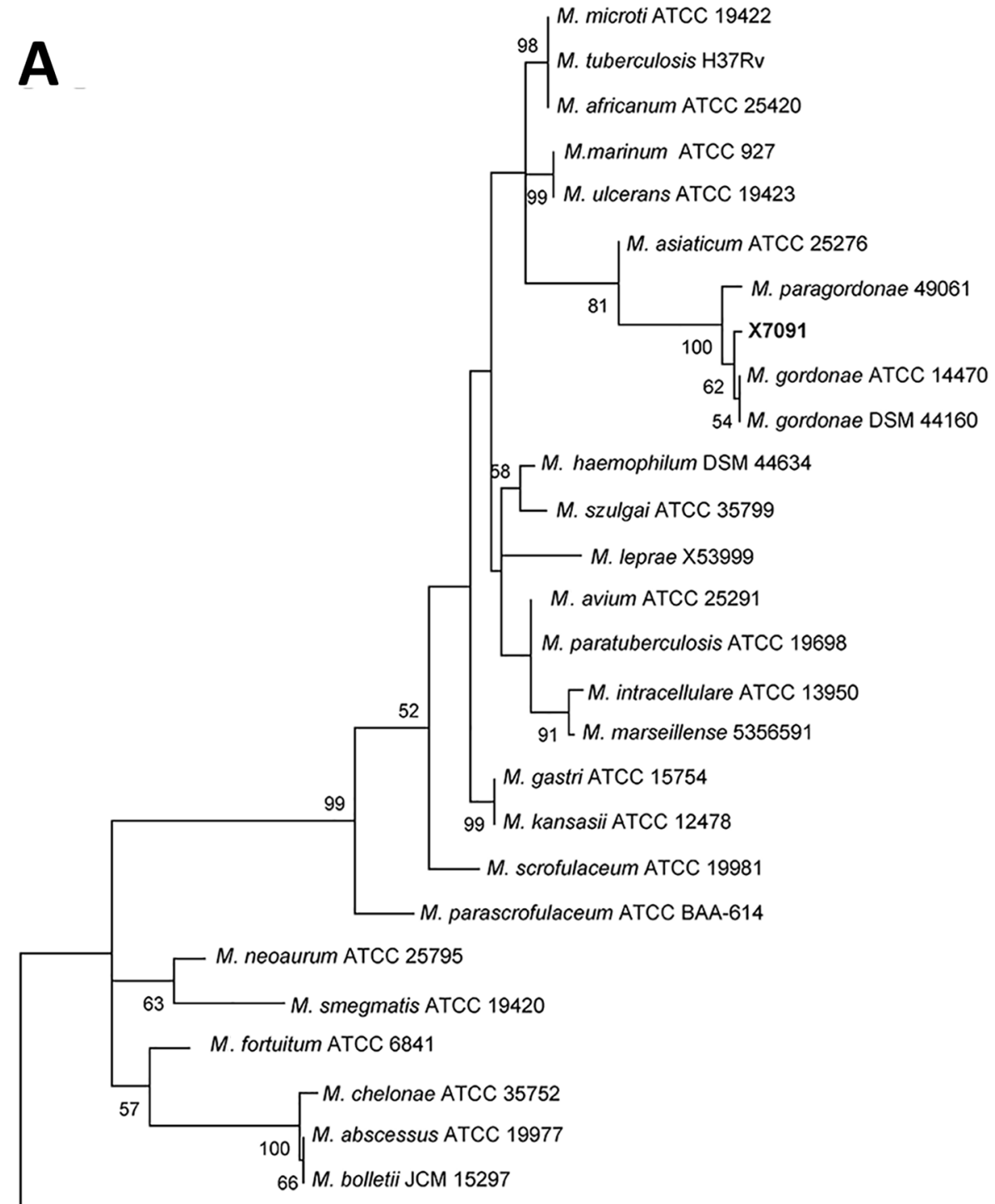

Figure 2. Phylogenetic trees of isolate from novel Mycobacterium gordonae-like infection in a 63-year-old man in China (X7091) and reference isolates. A) Evolutionary tree involving $16 \mathrm{~S}$ rRNA gene $(1,067$ positions) of isolate X7091 and 26 Mycobacterium strains. Tree constructed using the maximumlikelihood method and TamuraNei model with 500 bootstrap replications in MEGA X (https:// www.megasoftware.net). We selected Norcadia seriolae ATCC 43993 as the outgroup. B) Core genome-based maximumlikelihood phylogeny of isolate X7091 and other M. gordonaelike strains analyzed by Roary (https://sanger-pathogens.github. io/Roary) and constructed with a general time-reversible plus gamma maximum model (500 bootstrap replications) using the RaxML tool (14). We selected Mycobacterium marinum ATCC 927 as the outgroup. Scale bars indicate the number of nucleotide substitutions per site.

B

$\vdash_{0.01}$

Nocardia seriolae ATCC 43993

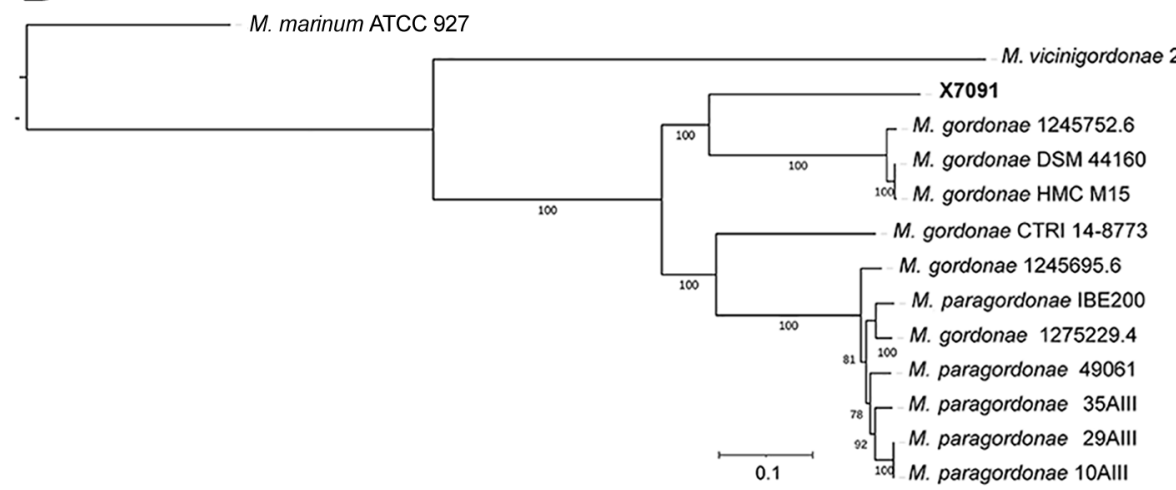


Conventional routines for describing species, such as wet-lab DDH and phenotypic tests, which are tedious and restricted by laboratory capacity, often do not delineate closely related species. Genomebased analysis affords a more accurate alternative for delineating species. Although digital gene-expression analyses might not have provided enough conclusive authentication data, the low genomewide similarity between the strain X7091 and M. gordonae group strongly support it as a novel species. Only a few $M$. gordonae assembly models are available, and largescale investigations are needed to better understand species diversity, geographic distribution, and clinical significance of M. camsnse sp. nov. infections.

The persistent but limited nonpurulent lesion of the immunosuppressed patient in our study reflects the attenuated nature of the pathogen. The various drug resistance properties of X7091 and other M. gordonae-like strains indicate the need for drug sensitivity testing before initiating drug treatment for M. gordonae-like strain infections $(6,7)$. Our patient responded well, indicating that operation combined with antimicrobial therapy could be a good option for treating environmental Mycobacterium-induced skin infections.

This study was supported by grants from the Chinese Academy of Medical Sciences Innovation Fund for Medical Sciences (2016-I2M-1-005, 2017-I2MB\&R-14) and the National Natural Science Foundation of China (81972950).

\section{About the Author}

Dr. Mei is a dermatologist at the Institute of Dermatology, Chinese Academy of Medical Sciences and Peking Union Medical College in Nanjing, China. Her primary research interest is mycobacterial infectious diseases.

\section{References}

1. Itoh S, Kazumi Y, Abe C, Takahashi M. Heterogeneity of RNA polymerase gene (rpoB) sequences of Mycobacterium gordonae clinical isolates identified with a DNA probe kit and by conventional methods. J Clin Microbiol. 2003;41:1656-63. https:/ / doi.org/10.1128/JCM.41.4.1656-1663.2003

2. Kim BJ, Hong SH, Kook YH, Kim BJ. Mycobacterium paragordonae sp. nov., a slowly growing, scotochromogenic species closely related to Mycobacterium gordonae. Int J Syst Evol Microbiol. 2014;64:39-45. https://doi.org/10.1099/ ijs.0.051540-0

3. Liu G, Yu X, Luo J, Hu Y, Dong L, Jiang G, et al. Mycobacterium vicinigordonae sp. nov., a slow-growing scotochromogenic species isolated from sputum. Int J Syst Evol Microbiol. 2021;71:71. https:/ / doi.org/10.1099/ ijsem.0.004796

4. Loret JF, Dumoutier N. Non-tuberculous mycobacteria in drinking water systems: a review of prevalence data and control means. Int J Hyg Environ Health. 2019;222:628-34. https://doi.org/10.1016/j.ijheh.2019.01.002

5. Barber TW, Craven DE, Farber HW. Mycobacterium gordonae: a possible opportunistic respiratory tract pathogen in patients with advanced human immunodeficiency virus, type 1 infection. Chest. 1991;100:716-20. https://doi.org/10.1378/chest.100.3.716

6. Al-Busaidi I, Wong D, Boggild AK. Cutaneous Mycobacterium gordonae infection in an elderly diabetic returned traveller. J Travel Med. 2017;24:24. https:/ / doi.org/10.1093/jtm/tax019

7. Chen Y, Jiang J, Jiang H, Chen J, Wang X, Liu W, et al. Mycobacterium gordonae in patient with facial ulcers, nosebleeds, and positive T-SPOT.TB test, China. Emerg Infect Dis. 2017;23:1204-6. https:// doi.org/10.3201/eid2307.162033

8. Cheung CY, Cheng NHY, Ting WM, Chak WL. Mycobacterium paragordonae: a rare cause of peritonitis in a peritoneal dialysis patient. Clin Nephrol. 2017;88:371-2. https:// doi.org/10.5414/CN109272

9. Takajo I, Iwao C, Aratake M, Nakayama Y, Yamada A, Takeda N, et al. Pseudo-outbreak of Mycobacterium paragordonae in a hospital: possible role of the aerator/ rectifier connected to the faucet of the water supply system. J Hosp Infect. 2020;104:545-51. https:/ / doi.org/10.1016/ j.jhin.2019.11.014

10. Jagielski T, Borówka P, Bakuła Z, Lach J, Marciniak B, Brzostek A, et al. Genomic insights into the Mycobacterium kansasii complex: an update. Front Microbiol. 2020;10:2918. https:// doi.org/10.3389/fmicb.2019.02918

11. Jain C, Rodriguez-R LM, Phillippy AM, Konstantinidis KT, Aluru S. High throughput ANI analysis of $90 \mathrm{~K}$ prokaryotic genomes reveals clear species boundaries. Nat Commun. 2018;9:5114. https://doi.org/10.1038/s41467-018-07641-9

12. Zong Z. Genome-based taxonomy for bacteria: a recent advance. Trends Microbiol. 2020;28:871-4. https://doi.org/ 10.1016/j.tim.2020.09.007

13. Meier-Kolthoff JP, Göker M. TYGS is an automated high-throughput platform for state-of-the-art genomebased taxonomy. Nat Commun. 2019;10:2182. https://doi.org/10.1038/s41467-019-10210-3

14. Stamatakis A. RAxML version 8: a tool for phylogenetic analysis and post-analysis of large phylogenies. Bioinformatics. 2014;30:1312-3. https:/ / doi.org/10.1093/ bioinformatics/btu033

15. Luo T, Xu P, Zhang Y, Porter JL, Ghanem M, Liu Q, et al. Population genomics provides insights into the evolution and adaptation to humans of the waterborne pathogen Mycobacterium kansasii. Nat Commun. 2021;12:2491. https://doi.org/10.1038/s41467-021-22760-6

Address for correspondence: Hong-sheng Wang, Institute of Dermatology, Chinese Academy of Medical Sciences and Peking Union Medical College, St.12 Jiangwangmiao, Nanjing, Jiangsu, 210042, China; email: whs33@vip.sina.com 